\title{
A Note on Set Systems with no Union of Cardinality 0 Modulo $m$
}

\author{
Vince Grolmusz \\ Department of Computer Science, Eötvös University, H-1117 Budapest, HUNGARY. \\ e-mail: grolmusz@cs.elte.hu
}

received September, 2001, revised October, 2002, accepted March, 2003.

Alon, Kleitman, Lipton, Meshulam, Rabin and Spencer (Graphs. Combin. 7 (1991), no. 2, 97-99) proved that for any hypergraph $\mathcal{F}=\left\{F_{1}, F_{2}, \ldots, F_{d(q-1)+1}\right\}$, where $q$ is a prime-power, and $d$ denotes the maximum degree of the hypergraph, there exists an $\mathcal{F}_{0} \subset \mathcal{F}$, such that $\left|\bigcup_{F \in \mathcal{F}_{0}} F\right| \equiv 0 \quad(\bmod q)$. The main tool of the proof was a one-to-one correspondence between hypergraphs and polynomials. We give a direct, alternative proof to this correspondence, and also review its implications for set-systems following from the result of Barrington, Beigel and Rudich (Comput. Complexity, 4 (1994), 367-382) for certain mod 6 polynomials.

Keywords: Set systems, composite modulus, polynomials over rings

\section{Introduction}

Alon, Kleitman, Lipton, Meshulam, Rabin and Spencer [1] gave the following definition:

Definition 1 ([1] ) For integers $d, m \geq 1$, let $f_{d}(m)$ denote the smallest $t$ such that for any hypergraph $\mathcal{F}=\left\{F_{1}, F_{2}, \ldots, F_{t}\right\}$ with maximum degree d there exists a non-empty $\mathcal{F}_{0} \subset \mathcal{F}$, such that $\left|\bigcup_{F \in \mathcal{F}_{0}} F\right| \equiv 0$ $(\bmod m)$

Baker and Schmidt [2] defined the following quantity:

Definition 2 For integers $d, m \geq 1$, let $g_{d}(m)$ denote the smallest $t$ such that for any polynomial $h \in$ $Z\left[x_{1}, x_{2}, \ldots, x_{t}\right]$ of degree $d$, satisfying $h(\mathbf{0})=0$, there exists an $\mathbf{0} \neq \varepsilon \in\{0,1\}^{n}$, such that $h(\varepsilon) \equiv 0$ $(\bmod m)$.

The following theorem was proven in [1]]:

Theorem 3 ([1] $]$ )

$$
f_{d}(m)=g_{d}(m)
$$

In the next section we give a natural one-to-one correspondence between polynomials and hypergraphs, proving Theorem 3 .

For $p$ prime, and $\alpha$ positive integer it is known ([1] ] [2], [4] ) that $g_{d}\left(p^{\alpha}\right)=d\left(p^{\alpha}-1\right)+1$, so we obtain 1365-8050 (c) 2003 Discrete Mathematics and Theoretical Computer Science (DMTCS), Nancy, France 
Corollary 4 ([[]]) For $\mathcal{F}=\left\{F_{1}, F_{2}, \ldots, F_{d(q-1)+1}\right\}$, where $q$ is a prime-power, and d denotes the maximum degree of the hypergraph, there exists an $\emptyset \neq \mathcal{F}_{0} \subset \mathcal{F}$, such that $\left|\bigcup_{F \in \mathcal{F}_{0}} F\right| \equiv 0 \quad(\bmod q)$.

This corollary is a generalization of the undergraduate exercise that from arbitrary $m$ integers, one can choose a non-empty subset, which adds up to 0 modulo $m$ (the $d=1$ case).

In 1991, Barrington, Beigel and Rudich [3] gave an explicit construction for polynomials modulo $m=$ $p_{1}^{\alpha_{1}} p_{2}^{\alpha_{2}} \ldots p_{r}^{\alpha_{r}}$, showing that

$$
g_{d}(m)=\Omega\left(d^{r}\right) .
$$

Since the proof of Theorem 3 (both the original and ours in the next section) gives explicit constructions for hypergraphs from polynomials, the following corollary holds:

Corollary 5 Let $m=p_{1}^{\alpha_{1}} p_{2}^{\alpha_{2}} \ldots p_{r}^{\alpha_{r}}$. Then there exists an explicitly constructible hypergraph $\mathcal{F}$ of maximum degree $d$, such that $|\mathcal{F}|=\Omega\left(d^{r}\right)$ and for each $\emptyset \neq \mathcal{F}_{0} \subset \mathcal{F}$ it is satisfied that $\left|\bigcup_{F \in \mathcal{F}_{0}} F\right| \not \equiv 0$ $(\bmod m)$.

The authors of [U] gave a doubly-exponential upper bound on $f_{d}(m)$, which was based on a Ramseytheoretic bound of [2]. More recently, Tardos and Barrington [4] showed that

$$
f_{d}(m)=\exp \left(O\left(d^{r-1}\right)\right) .
$$

\section{Correspondence between polynomials and hypergraphs}

We give here a short and direct proof for Theorem 3. Let $Q$ denote the set of rationals. It is well known that the set of functions $\left\{f:\{0,1\}^{t} \rightarrow Q\right\}$ forms a $2^{t}$-dimensional vector space over the rationals. One useful basis of this vector space is the set of OR-functions $\left\{\bigvee_{i \in I} x_{i}: I \subset\{1,2, \ldots, t\}\right\}$, where

$$
\bigvee_{i \in I} x_{i}=1-\prod_{i \in I}\left(1-x_{i}\right) .
$$

It is easy to see that any integer-valued function on the hypercube can be written as the integer-coefficient linear combination of these OR-functions. Moreover, if the function is a degree- $d$ polynomial, then it is enough to use OR functions with $|I| \leq d$. If we consider modulo $m$ polynomials, then the coefficients can be restricted to the set $\{0,1,2, \ldots, m-1\}$. It will be convenient to view modulo $m$ polynomials as the sum of several OR functions with coefficient 1; instead of multiplying an OR function with a coefficient $a$ we will add it up exactly $a$ times.

Consequently, our degree- $d$ modulo $m$ polynomial has the following form:

$$
h=S_{1}+S_{2}+\cdots+S_{\ell},
$$

where $S_{i}$ is an OR-function of degree at most $d$.

Now we are ready to define the one-to-one correspondence between degree- $d$ modulo $m$ polynomials without non-trivial zeroes on the hypercube and hypergraphs, without non-empty subhypergraphs of modulo- $m$ union-size 0 . Let $h$ be a degree- $d$ polynomial written in form (1), and define hypergraph $\mathcal{F}=\left\{F_{1}, F_{2}, \ldots, F_{t}\right\}$, where $F_{i}=\left\{S_{j}: x_{i}\right.$ appears as a variable in $\left.S_{j}\right\}$. Clearly, the degree of this hypergraph is at most the degree of $h$ that is, $d$. 
On the other hand, for a hypergraph $\mathcal{F}=\left\{F_{1}, F_{2}, \ldots, F_{t}\right\}$ on the ground-set $\left\{v_{1}, v_{2}, \ldots, v_{\ell}\right\}$, let us define $h\left(x_{1}, x_{2}, \ldots, x_{t}\right)=S_{1}+S_{2}+\cdots+S_{\ell}$, where

$$
S_{j}=\bigvee_{i: v_{j} \in F_{i}} x_{i}
$$

Obviously, the degree of $h$ is at most the degree of $\mathcal{F}$.

Now we state that $\mathcal{F}$ has a non-empty subhypergraph with union-size 0 modulo $m$ if and only if there exists a $\mathbf{0} \neq \mathbf{x}: h(\mathbf{x}) \equiv 0 \quad(\bmod m)$. The proof is as follows: For $\mathbf{x}=\left(x_{1}, x_{2}, \ldots, x_{n}\right) \in\{0,1\}^{n}$ let us denote $I(\mathbf{x})=\left\{i: x_{i}=1\right\}$. Then $S_{j}(\mathbf{x})=1$ if $S_{j} \in \bigcup_{i \in I(\mathbf{x})} F_{i}$, and $S_{j}(\mathbf{x})=0$ otherwise. Thus $h(\mathbf{x})=\left|\bigcup_{i \in I(\mathbf{x})} F_{i}\right|$ holds for all $\mathbf{x} \in\{0,1\}^{n}$. In particular, evaluations of $h$ and union-sizes of subhypergraphs in $\mathcal{F}$ become divisible by $m$ simultaneously.

\section{Acknowledgment.}

The author is grateful for the anonymous referee for his/her suggestions clarifying the correspondence between polynomials and hypergraphs. The author also acknowledges the partial support of research grants EU FP5 IST FET No. IST-2001-32012, OTKA T030059. 


\section{References}

[1] N. Alon, D. Kleitman, R. Lipton, R. Meshulam, M. Rabin, and J. Spencer. Set systems with no union of cardinality 0 modulo m. Graphs and Combinatorics, 7:97-99, 1991.

[2] R. Baker and W. Schmidt. Diophantine problems in variables restricted to the values 0 and 1. Journal of Number Theory, 12:460-486, 1980.

[3] D. A. M. Barrington, R. Beigel, and S. Rudich. Representing Boolean functions as polynomials modulo composite numbers. Comput. Complexity, 4:367-382, 1994. Appeared also in Proc. 24th Ann. ACM Symp. Theor. Comput., 1992.

[4] G. Tardos and D. A. M. Barrington. A lower bound on the MOD 6 degree of the OR function. Comput. Complexity, 7:99-108, 1998. 